\title{
La generación de ambientes de aprendizaje: un análisis de la percepción juvenil
}

\author{
The generation of learning environments: an analysis of the perception of \\ youth
}

Ambientes de aprendizagem geração: uma análise da percepção juvenil

DOI: http://dx.doi.org/10.23913/ride.v7i14.276

Leonor Antonia Espinoza Núñez

Universidad Autónoma de Sinaloa, México

leonor_espinoza63@hotmail.com

René Rodríguez Zamora

Universidad Autónoma de Sinaloa, México

rene.rodriguez@info.uas.edu.mx

\section{Resumen}

En este artículo se presenta un análisis cuyo objetivo es identificar las características del ambiente de aprendizaje desde la percepción de los jóvenes estudiantes de bachillerato a partir de las necesidades escolares que garantizan el rendimiento académico y el desarrollo de competencias, considerando como principales atributos: , la interacción social, la normatividad, los recursos y la práctica docente.

El ambiente de aprendizaje entendido en su complejidad sumando todos los componentes que lo integran, desde sus actores principales maestro y alumno y las actividades que desarrollan en una dinámica de interacción, las estrategias y los recursos que se utilizan para involucrar el contexto partiendo del conocimiento previo, el construir y compartir objetivos métodos y planes, todo ello con la intención de generar nuevo conocimiento, requiere una visión que trasciende el espacio áulico, refiere al mundo de la interacción del sujeto que aprende con su contexto social y con su propia habilidad para entender el mundo. 
Se realizó un estudio descriptivo explicativo con un enfoque cuantitativo y cualitativo considerando una muestra representativa de 267 jóvenes de bachillerato universitario. Se encontró que la sociohabilidad entre coetáneos es una de las características más importantes para la generación de ambientes de aprendizaje, que no ha sido considerada por las figuras que representan a las autoridades institucionales educativas, en torno a estas se mostró una insatisfacción en su vínculo de comunicación directa y confianza, a mayor rango de responsabilidad menor contacto directo hay con los estudiantes. El contacto con sus autoridades implica la oportunidad de sentirse atendido y sobre todo comprometido en la aplicación de la norma, ello le fortalece además su sentido de pertenencia e identidad con la institución. Con respecto a la normatividad se observó en los resultados que es entendida como mecanismo de control sin un respectivo seguimiento, una práctica docente cuyas estrategias están desvinculadas al contexto cotidiano en el que pueda enmarcar un aprendizaje útil y con sentido. Finalmente los resultados de este estudio pueden servir como referente para que las autoridades educativas consideren el potencial que representa el aspecto de la socialización de los jóvenes en las diferentes dimensiones que conforman las condiciones para crear ambientes de aprendizaje acordes y pertinentes a las demandas estudiantiles.

Palabras clave: Ambiente de aprendizaje, Bachillerato, Percepción Juvenil, Práctica Docente, Sociohabilidad.

\section{Abstract}

This article presents an analysis which aims to identify the characteristics of the learning environment from the perception of young high school students from the school needs that ensure academic achievement and the development of competencies, whereas as main attributes: social interaction, regulations, resources and teaching practice.

El ambiente de aprendizaje entendido en su complejidad sumando todos los componentes que lo integran, from the lead actors teacher and student and the activities carried out in a dynamic of interaction, strategies and resources that are used to involve the context based on the prior knowledge, build and share goals methods and plans, all with the aim of creating new knowledge, requires a vision that transcends the grandiose space, refers to the world of the interaction of the subject learning with their social context and its own ability to understand the world. 
We performed a descriptive explanatory study with a quantitative and qualitative approach whereas a representative sample of 267 youth from University high school. We found that the sociohabilidad between peers is one of the most important features for the generation of learning environments, which has not been considered by the figures that represent educational institutional authorities, around these dissatisfaction expressed in its bond of confidence, a greater range of liability less direct contact and direct communication with the students. Contact with their authorities implies the opportunity to feel cared and above all committed to the application of the standard, it strengthens him also their sense of belonging and identity with the institution. With respect to the regulations was observed in the results is understood as a mechanism of control without respective monitoring, a practice whose strategies are unrelated to the everyday context in which to frame a useful learning and sense. Finally the results of this study can serve as a reference so that the educational authorities consider the potential that represents the appearance of the socialization of young people in the different dimensions that make up the conditions to create learning environments that are consistent and relevant to the student demands.

Key words: learning environment, high school, youth perception, teaching practice, socioability.

\section{Resumo}

Este artigo apresenta uma análise teve como objetivo identificar as características do ambiente de aprendizagem a partir da percepção de jovens estudantes do ensino médio de escola precisa para garantir a realização e desenvolvimento de habilidades acadêmicas, considerando como atributos principais é apresentado: o interação social, regulamentos, recursos e prática de ensino.

O ambiente de aprendizagem compreendido em sua complexidade, adicionando todos os componentes que compõem o que, desde o seu principal professor atores e aluno e suas atividades em uma interação dinâmica, estratégias e recursos utilizados para envolver o contexto baseado em conhecimentos prévios a construção e partilha de métodos e planos objetivos, tudo com a intenção de gerar novos conhecimentos, requer uma visão que transcende o espaço da sala de aula, refere-se ao mundo da interação do aluno com seu contexto social e sua própria capacidade de compreender o mundo. 
um estudo descritivo explicativo com uma abordagem quantitativa e qualitativa, considerando uma amostra representativa de 267 bacharelado universitário novo foi realizada. Verificou-se que sociohabilidad entre pares é um dos ambientes de aprendizagem geração mais importantes, que não foi considerado pelas figuras que representam as autoridades institucionais educacionais sobre esses recursos insatisfação ele estava em seu elo comunicação direta e de confiança, uma gama maior de responsabilidade menos contato direto com os alunos lá. O contato com as autoridades implica a oportunidade de se sentir cuidado e especialmente comprometido com a implementação da norma, que também irá reforçar o seu sentido de pertença e de identidade com a instituição. No que diz respeito à regulação observada nos resultados entende-se como um mecanismo de controlo sem respectiva pista, uma prática de ensino cujo estratégias são dissociados de contexto diária que pode moldar uma aprendizagem útil e significativo. Finalmente, os resultados deste estudo podem servir como uma referência para que as autoridades educacionais considerar o potencial do aspecto da socialização dos jovens nas diferentes dimensões que compõem as condições para a criação de ambientes de aprendizagem acordes e relevantes para as demandas dos alunos.

Palavras-chave: ambiente de aprendizagem, bacharelado, Percepção Juventude, Prática de Ensino, Sociohabilidad.

Fecha Recepción: Junio 2016 Fecha Aceptación: Diciembre 2016

\section{Introducción}

El desarrollo de competencias, enfoque de la vigente Reforma Integral de la Educación Media Superior en México (RIEMS, 2008), tiene sus principios fundamentales en la psicología del aprendizaje y en la sociología, entre otras disciplinas, y parte de la teoría constructivista aún con una perspectiva distinta, en palabras de Tobón, Prieto y Fraile (2010) transitando de la lógica de los contenidos a la lógica de la acción. En esta perspectiva el aprendizaje está basado en la actividad cognitiva que el sujeto puede gestionar en su propio contexto con el apoyo y orientación de un facilitador. Se pretende desde este marco formador articular el ámbito social y productivo con el ámbito educativo bajo las premisas que marca las necesidades de la sociedad moderna. 
En este sentido, es necesario replantearse la actividad docente, pues la transmisión del conocimiento deja de ser la responsabilidad principal de la formación del educando, se exige un cambio en la visión y expectativa de todos los involucrados pero principalmente un cambio de actitud que se manifieste en asumir un nuevo rol, mediante una planeación que conlleve a la generación de verdaderos ambientes de aprendizaje. Ambientes donde el estudiante encuentre las condiciones para participar en su proceso de formación.

El ambiente de aprendizaje incluye y supera las condiciones físicas y de infraestructura y recursos, que si bien son indispensables serían insuficientes en sí mismos. Se requiere, tarea fundamental del profesor como mediador o facilitador generar un clima social que propicie la sana y asertiva relación y el establecimiento de vínculos directivos/alumnos, profesor-/alumno, alumno/alumno, pues en esta habilidad social residen las condiciones del aprendizaje autónomo y colaborativo para lograr la potencialización de las habilidades de los jóvenes y con ello garantizar el desarrollo de competencias.

En este estudio se presenta un análisis sobre los ambientes de aprendizaje generados a partir de las necesidades y percepciones de los jóvenes para el aprendizaje significativo y el desarrollo de competencias en el bachillerato, en aras de que esto sirva en la elaboración de propuestas que permitan propiciar condiciones que conduzcan a la mejora de la acción educativa.

\section{MÉTODO}

Para la realización del estudio se tomó de una población de más de 2000 sujetos una muestra aleatoria estratificada de 267 estudiantes del nivel medio superior pertenecientes a una sola institución escolar cuyas edades oscilan entre los 15 y 18 años. La recolección de datos se realizó mediante un cuestionario elaborado a partir de la variable principal: Ambientes de Aprendizaje y la descripción de sus atributos, conformando a partir de ellos nuevas varibles: Interacción Social, Normatividad, Infraestructura, y Práctica Docente. Dicho cuestionario fue validado utilizando el estadístico Alpha de crombach. Como parte del procesamiento se abordó el análisis descriptivo utilizando principalmente las medidas de tendencia central, y el cálculo de frecuencias y porcentajes, obteniendo a partir de ello la caracterización de la muestra con respecto al fenómeno estudiado. Para cada agrupación de variables se consideró la media de medias $X x$ y su respectiva $\mathrm{S}$ para establecer los límites superior e inferior, mediante la fórmula $(X x \pm S)$. A partir de ello se obtiene información de las variables que muestran relevancia. 
Con el fin de obtener la relación entre las variables se aplicó el cálculo estadístico de correlación, con una carga factorial mínima $\mathrm{r} \geq .16$, y un nivel de probabilidad $\mathrm{p} \leq .01$. Se utilizó además la prueba $T$-Student para obtener datos comparativos estableciendo diferencias entre grupos.

\section{AMBIENTES DE APRENDIZAJE}

Respecto al concepto, ambiente de aprendizaje, de acuerdo a Duarte (2003), está relacionado con la idea geográfica para referirse al entorno o medio, sin embargo se consideró que la palabra medio era insuficiente para designar la acción del ser humano sobre ese entorno por lo que construyeron el concepto de ambiente, el cual involucra al entorno y la acción de los seres humanos sobre él.

El ambiente debe trascender, entonces, la noción simplista de espacio físico, como contorno natural y abrirse a las diversas relaciones humanas que aportan sentido a su existencia. Desde esta perspectiva se trata de un espacio de construcción significativa de la cultura. De esta manera el ambiente de aprendizaje se entiende como el entorno o el contexto natural al interior del cual se producen relaciones humanas que forman parte del hecho educativo.

Hablar de ambiente de aprendizaje y desarrollo de competencias tal como lo plantea la RIEMS es hablar de que los docentes tienen que transformar su práctica de enseñanza tradicionalista al modelo constructivista, ya que el enfoque de competencias requiere que éstas sean movilizadas en la práctica del estudiante en su contexto, en tanto que el modelo constructivista se caracteriza porque el conocimiento se construye vinculándose a las situaciones y problemas en que se desenvuelve el estudiante.

Una de las principales características del ambiente escolar desde el modelo constructivista es que el docente centra su atención en la actividad cognitiva del estudiante, y debe propiciar condiciones para que los estudiantes construyan sus propios significados, comenzando con las creencias, los conocimientos y las prácticas culturales que traen al salón de clases para poder lograr el aprendizaje significativo.

En el mismo sentido, Cano y Lledó (1995) proponen los siguientes principios para un apropiado ambiente de aprendizaje: 1 . Facilitar que todas las personas del grupo se conozcan para crear un grupo cohesionado con objetivos y metas comunes. 2. Proporcionar a todos el contacto con materiales y actividades diversas que permitan abarcar un amplio abanico de aprendizajes cognitivos, afectivos y sociales. 3. Ha de ser diverso, debiendo trascender la idea de que todo 
aprendizaje se desarrolla entre las cuatro paredes del aula. 4. Ofrecer distintos subescenarios de tal forma que las personas del grupo puedan sentirse acogidas, según distintos estados de ánimo, expectativas e intereses. 5. Ha de ser construido activamente por todos los miembros del grupo y la escuela, viéndose en él reflejadas sus peculiaridades y su propia identidad.

Biggs (2006) habla del clima de clase al cual se refiere como la atmosfera que se crea en el salón de clase y que puede ser autoritaria, amistosa, fría o cálida.

Bolaños y Molina (2007) señalan que el ambiente de aprendizaje no debe restringirse al aula escolar y es necesario incorporar a este concepto cualquier elemento en el cual interactúen alumnos y docentes. Señalan que este tipo de ambiente debe propiciar aprendizajes motivantes y permanentes, por lo que alumnos y docentes deben cooperar para lograr un ambiente físico apropiado y un ambiente afectivo donde se promuevan la libertad de expresión, el respeto, la solidaridad y la democracia.

Al mismo respecto Gimeno y Pérez (2008) aseveran que el ambiente de aprendizaje es un entorno socio-psicológico y material donde los estudiantes y los docentes trabajan juntos. Las relaciones que se establecen en este medio representan una red de variables culturales, psicológicas, institucionales y sociales. El tipo de interacción que se establece en esta red es lo que le da un carácter especial a cada ambiente de aprendizaje.

Usando categorías parecidas, Caso, Salgado, Rodríguez, Contreras y Urias (2010) hablan del clima escolar como el entorno de aprendizaje donde se establecen relaciones entre docentes, alumnos y personal administrativo, donde hay crecimiento personal y claridad en las normas escolares. Aseveran que es donde se da la convivencia y el trabajo colectivo, donde las relaciones entre alumnos-alumnos y docentes-alumnos cobran especial importancia por ser un espacio donde todos se enriquecen mutuamente. Parra (2007) afirma que un ambiente de aprendizaje constituye el conjunto de todos los recursos físicos y simbólicos del entorno que se pueden utilizar para lograr los procesos de aprendizaje más eficientes.

Como se ha explicado anteriormente el ambiente de aprendizaje abarca desde el entorno que comprende la parte física que rodea al estudiante como son aulas, jardines, laboratorios, instalaciones deportivas, hasta el tipo de relación que se establece entre estudiantes, docentes y directivos en búsqueda de lograr mejores aprendizajes. 
En este estudio y para fines de análisis de resultados se consideró el ambiente de aprendizaje a partir de cuatro categorías:

1. Interacción social: Comprende la relación entre compañeros, la relación con sus maestros y la relación con las autoridades escolares.

2. Normatividad: Incluye todo lo relacionado con las reglas escolares.

3. Instalaciones, recursos y herramientas.

4. Práctica docente que abarca: estrategias de enseñanza-aprendizaje y estrategias de evaluación.

\section{RESULTADOS}

\section{INTERACCIÓN SOCIAL}

En este apartado se describe y analiza todo lo que se refiere a cómo los estudiantes distinguen los diferentes tipos de relación que se establecen entre ellos como alumnos, entre alumnos y maestros y entre alumnos y autoridades.

Figura 1. Percepción de los estudiantes en cuanto a su interacción social en la escuela.

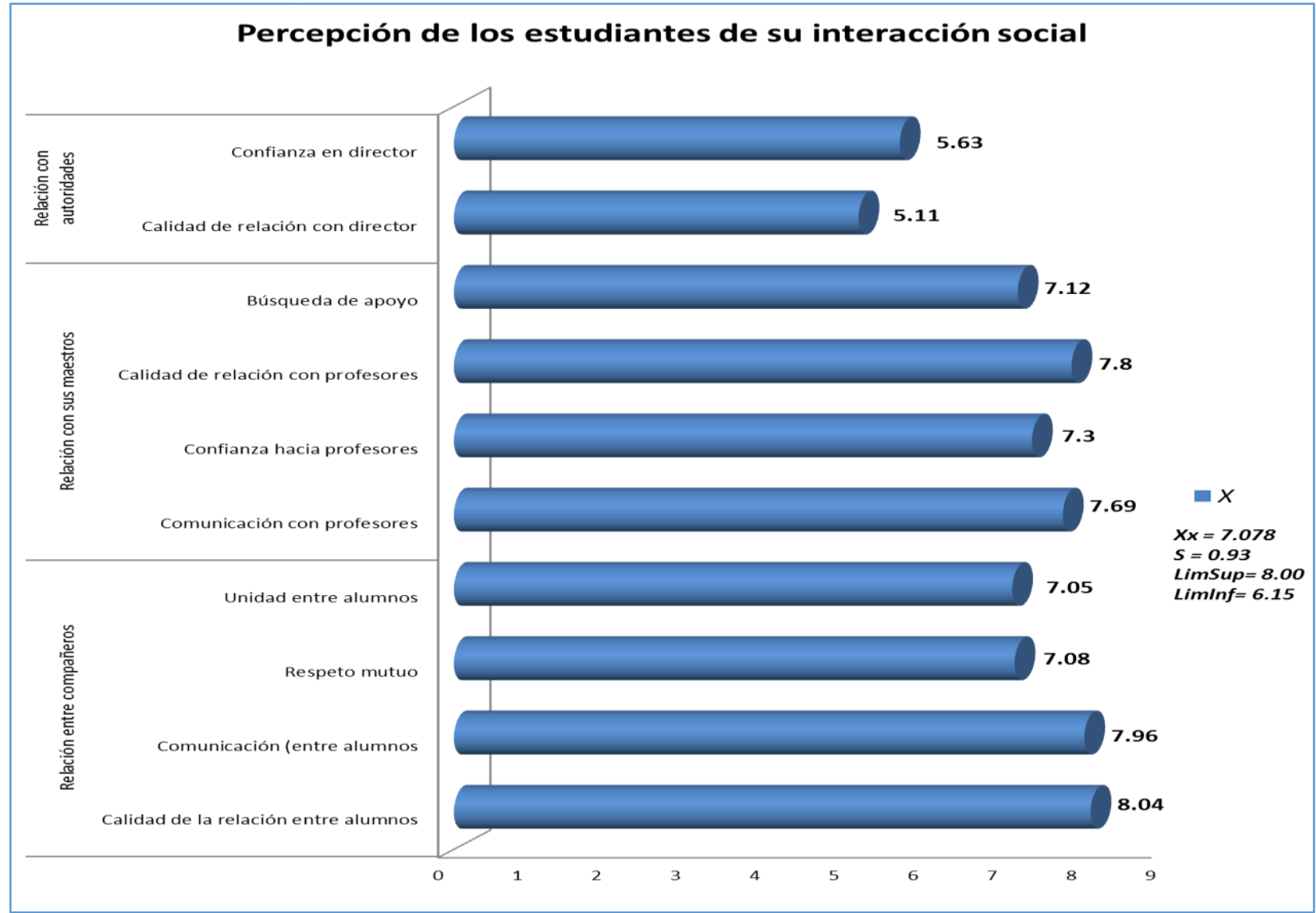

Fuente: Elaboración propia. 
Los datos reportan que la prioridad del estudiante reside en la calidad de la relación que establece entre sus compañeros, mostrando una gran satisfacción de la habilidad comunicativa que desarrolla entre sus pares. Esto indica que las buenas relaciones y el compañerismo entre los jóvenes de la escuela son una habilidad o competencia característica que genera condiciones para un aprendizaje colaborativo.

Los estudiantes comentan que aunque hay relaciones de amistad que dependen de la diversidad del tipo de alumno, el ambiente generalmente se distingue por un clima de camaradería, armonía, y mucha convivencia entre todos los miembros de un grupo o salón de clases, reconocen que el abuso o maltrato tipificado como bullying no es un circunstancia característica de esta escuela, pues las relaciones se basan en el respeto mutuo.

De acuerdo a los datos (ver Figura 1) la interacción entre los jóvenes estudiantes muestra el desarrollo de la sociohabilidad como una competencia altamente lograda, lo que implica que se construye un clima escolar favorable para promover el aprendizaje colaborativo, ya que muestran disposición a compartir a través de la convivencia mediante valores aceptables para asegurar la armonía en grupo.

Por otra parte, como atributos de significancia baja son las que refieren a la relación y confianza en el director y las autoridades de la escuela, esto sugiere que la relación o vínculo con las autoridades es insatisfactoria. A partir de ello el estudiante expresa que requiere de mayor acercamiento con las figuras institucionales, para desarrollar un sentido de identidad y pertenencia a la institución.

Del anterior reporte se puede inferir que a mayor rango de responsabilidad de las autoridades escolares menor contacto directo hay con los estudiantes. Para ellos el contacto con sus autoridades implica la oportunidad de sentirse atendido y sobre todo comprometido en la aplicación de la norma, ello le fortalece además su sentido de pertenencia e identidad con la institución.

Es un clima de sociohabilidad que está poco aprovechado por las autoridades institucionales para orientar las condiciones a la promoción de ambientes de aprendizaje apoyados inclusive en las tecnologías, factor que los estudiantes demandan como una de sus prioridades.

El clima de aprendizaje Vite (2014) lo define como la interacción, la comunicación entre los que se encuentran dentro del proceso de enseñanza - aprendizaje, esta debe darse entre el docente y los alumnos y viceversa, así como alumno - alumno. Dentro de este clima debe prevalecer la 
armonía, confianza, seguridad, respeto, para que los educandos con toda libertad puedan expresarse, dar a conocer alguna inquietud o duda a favor de la obtención de un verdadero aprendizaje.

De esta manera, las relaciones interpersonales que los jóvenes establecen entre si se convierte en un aspecto básico de sus vidas, funcionando además como medio para alcanzar los objetivos académicos, por lo que promover que esas relaciones se potencialicen y se orienten puede convertirse en una estrategia que el docente debe aprovechar para facilitar el aprendizaje.

Giraldo y Ortiz (2009) encontraron en su estudio que los estudiantes que participan de actividades que les permiten interacción fuera del ámbito escolar, especialmente con adultos y con pares, demuestran una mayor habilidad social para establecer y mantener relaciones efectivas que son la base de las competencias laborales generales. Aunque en este apartado se refiere a la interacción al interior del ambiente escolar, muestran también que dichas relaciones generan la autoconfianza para motivar su desempeño.

Con respecto a la relación del alumno con el docente como autoridad educativa, Medina (2015) afirma que la relevancia del clima motivacional que los profesores crean en el aula, reside en que permite a los alumnos saber qué es lo que importa en las clases, qué es lo que el docente quiere lograr con ellos y qué efectos puede tener en el aula de clases actuar de un modo u otro.

\section{NORMATIVIDAD}

En este apartado se describe cómo los estudiantes distinguen la parte del ambiente de aprendizaje que corresponde a la existencia de una normatividad que rija las relaciones escolares y su nivel de cumplimiento a las mismas. 
Figura 2. Percepción de los estudiantes en cuanto a la normatividad.

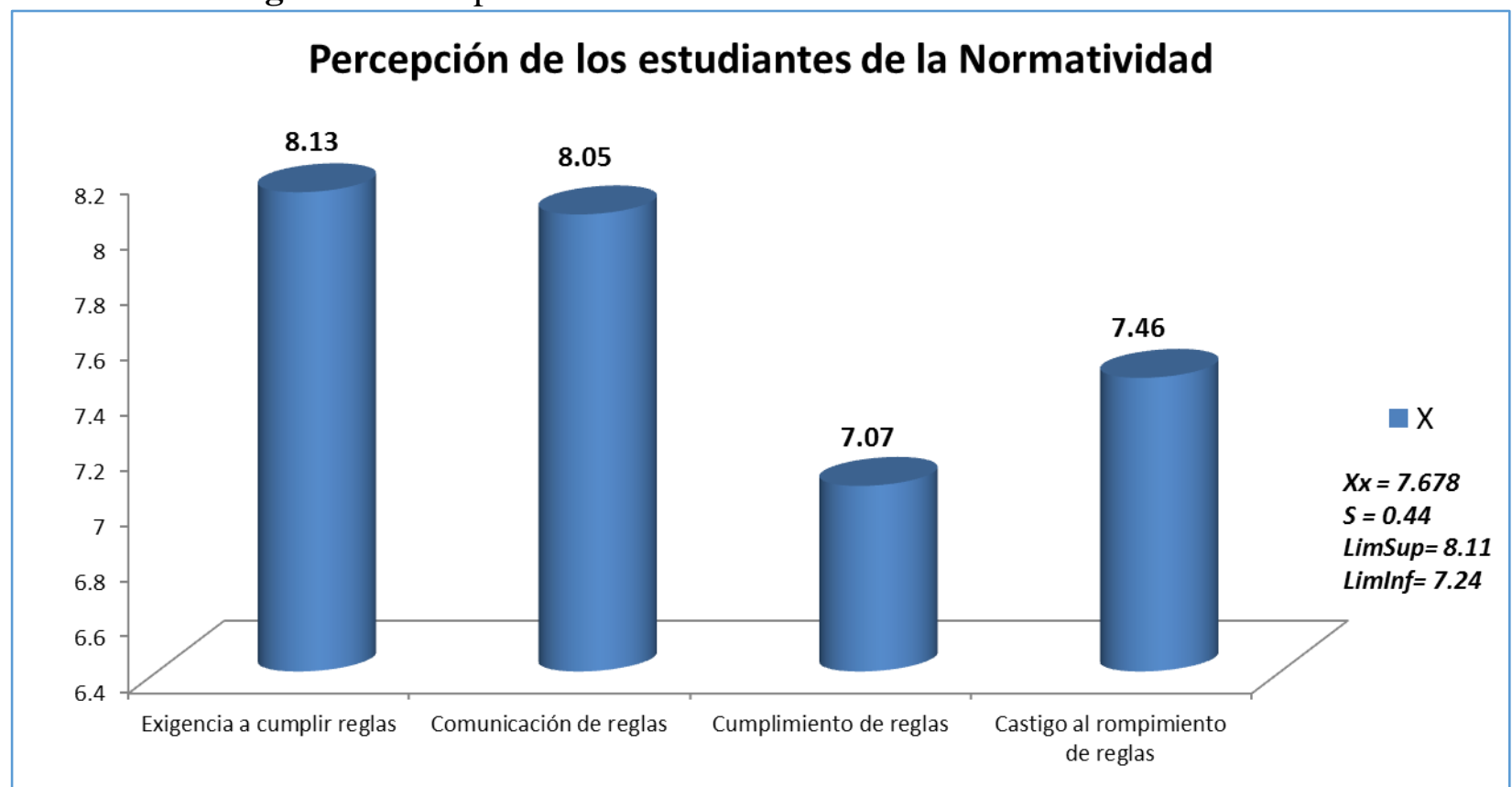

Fuente: Elaboración propia.

De acuerdo al comportamiento de esta agrupación de variables, los datos muestran (ver figura 2) que no hay una percepción muy aceptable con respecto a la manera en que se regula la vida académica al interior del clima escolar. Por un lado hay una exigencia al cumplimiento de normas mediante el ejercicio o ejecución de un reglamento establecido, sin darle el respectivo seguimiento a la implicación, pues la función está concentrada en la difusión o socialización del mismo y la implementación de lo más elemental y visible. En lo que respecta a los jóvenes estudiantes, hay reconocimiento en lo que refiere a la falta de cumplimiento de la normatividad, es decir los jóvenes aceptan que no siempre cumplen con las reglas que rigen en su escuela. Esto es congruente con el carácter rebelde de los adolescentes que se destaca por su enfrentamiento y desobediencia a las reglas impuestas por las autoridades, ya que comentan que no pasa nada si se llega al incumplimiento de las reglas establecidas por la falta de seguimiento a la disciplina escolar.

Esto habla de una falta de credibilidad en la función normativa que recae por una parte en la responsabilidad de las figuras institucionales y por otra, a la conducta consiente de los alumnos. Refiere además a la representación mental que el estudiante ha desarrollado hacia su institución, al no haber identidad, participación y claridad sobre el objetivo de la norma, no se desarrolla tampoco un adecuado nivel de compromiso. 
Como parte de la administración escolar, la estructura normativa ha de coadyuvar a la labor educativa asegurando la participación de todos los implicados bajo lineamientos institucionales que aseguren la participación asertiva de todos los implicados, generando un ambiente de convivencia y de desarrollo en el logro de los objetivos y metas que beneficien principalmente a la formación del estudiante, sin embargo, se observa en los resultados que existe una percepción de un clima social que no es regulado por las autoridades en el marco de la convivencia y el cumplimiento a las indicaciones escolares.

El problema de las normas, el orden y la disciplina según Dueñas (2015) es que la disciplina en la cultura escolar, más que entenderse como una disposición del discípulo para aprender, es un concepto asociado al orden, control y sanción del comportamiento de los alumnos. Idea que ha llevado a la escuela a instituir reglamentos y dispositivos de control para los estudiantes. El modelo basado en la emisión de reglamentos, está dirigido exclusivamente a controlar y sancionar los comportamientos de los estudiantes, su contenido y características obedecen a un modelo de gestión de la escuela caracterizado por el verticalismo y autoritarismo, si a este respecto se le suma la falta de cordialidad y comunicación entre las figuras de autoridad y confianza en la administración, la falta de involucramiento y de identidad en la solución de conflictos genera climas donde no se promueven hábitos orientados al aprovechamiento escolar.

\section{INSTALACIONES, RECURSOS Y HERRAMIENTAS}

En este apartado se describe el grado de satisfacción y demanda que los estudiantes tienen con respecto a las condiciones físicas, instrumentales y de equipamiento tecnológico que su institución les brinda para la generación de ambientes de aprendizaje. 
Figura 3. Percepción de los estudiantes en cuanto a la normatividad.

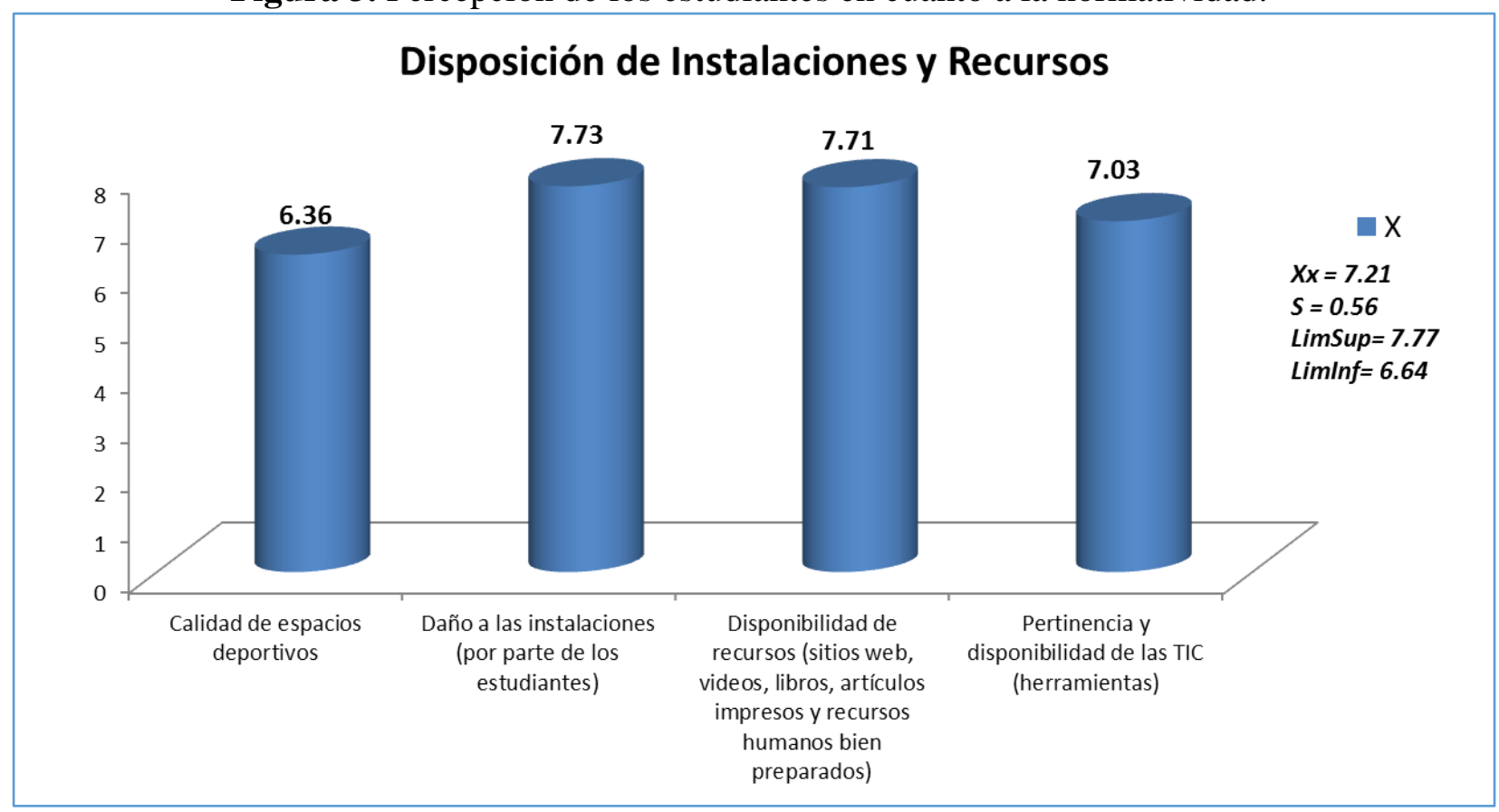

Fuente: Elaboración propia.

Como se puede observar en la figura 3, en esta agrupación no se muestran valores altos, lo que implica que no demostraron estar muy satisfechos de las condiciones de recursos que la institución les brinda y el cuidado que la población escolar hace de su uso. En lo que respecta a la disponibilidad para la utilización de espacios deportivos y recreativos, manifestaron sentir que son insuficientes y de poca calidad, de lo que se puede inferir que los estudiantes, demandan de condiciones en las que pueda socializar y ejercer actividades físicas donde pueda mejorar su calidad de vida además de tener una opción para el disfrute personal y fortalecer de manera sana y asertiva su relación con los demás. Para los jóvenes, el hecho de no contar con instalaciones ni programas que motiven a la práctica del deporte y la convivencia puede resultar contraproducente en la formación y establecimiento de hábitos asertivos y la prevención de conductas de riesgo.

Por otra parte la utilización de las tecnologías representa para ellos una de las principales demandas para abrirse a la socialización, es decir obedece a una necesidad social, y representa además una expectativa pedagógica, además de que se convierte en requisito cuya satisfacción le brinda mayor seguridad para vincularse con el sector laboral, en este sentido la demanda del estudiante es mayor que la forma en que la institución le contribuye, ya que declaran que la 
existencia, la pertinencia así como la utilización de herramientas tecnológicas como acceso a sitios web, plataformas educativas, y disponibilidad de herramientas diversas es apenas aceptable. En este caso para los jóvenes adolescentes, la infraestructura tecnológica se convierte en el medio socializador por excelencia, además de herramienta para el aprendizaje colaborativo, por ello la demanda se incrementa en este ámbito como lo muestra los resultados de este estudio.

\section{PRÁCTICA DOCENTE}

En este apartado se analiza la práctica docente constituida en actividades que comprenden el diseño e implementación de estrategias de enseñanza-aprendizaje y estrategias de evaluación.

Figura 4. Percepción de los alumnos en relación con el trabajo de sus maestros.

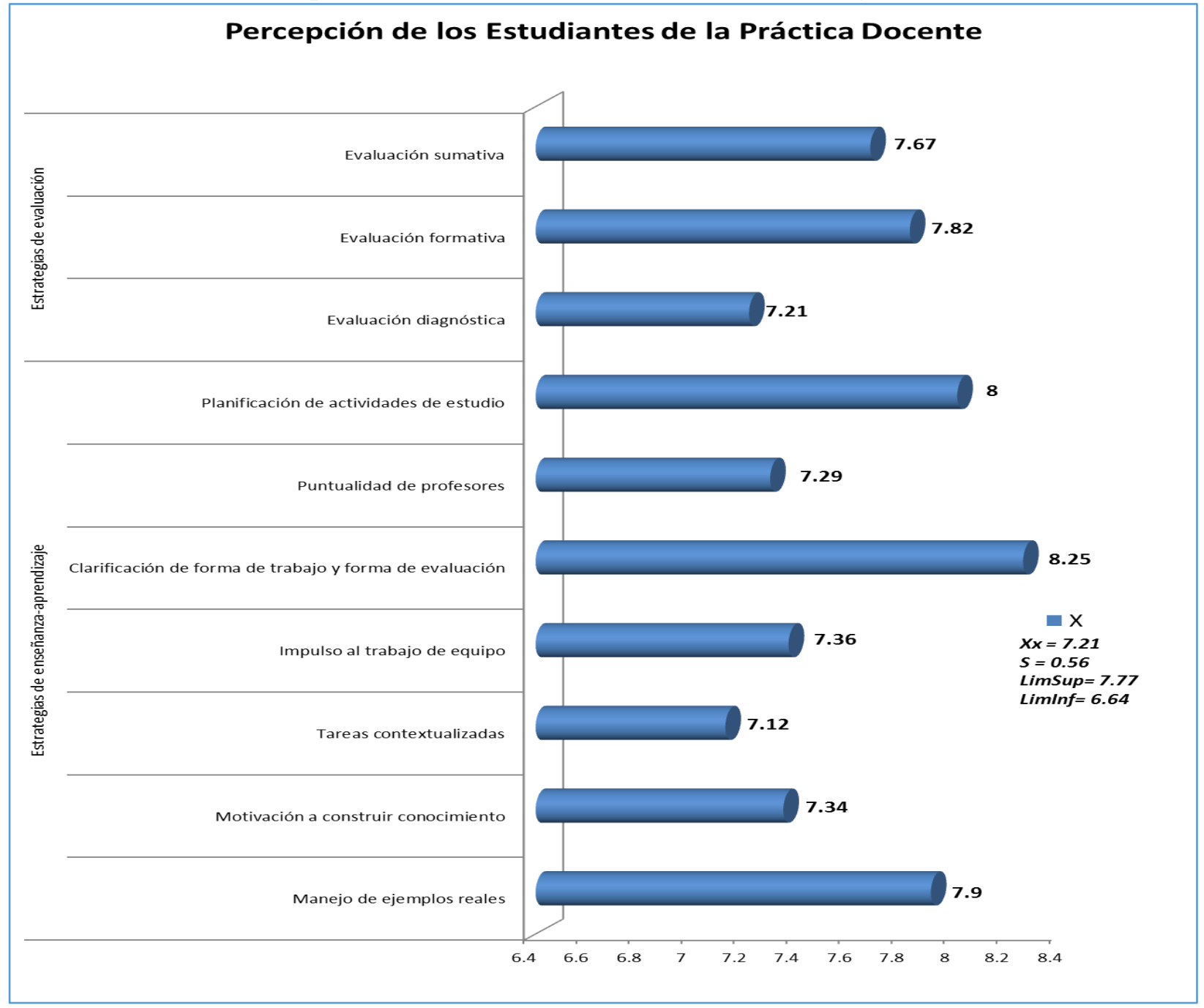

Fuente: Elaboración propia. 
Como se observa en la figura 4, los estudiantes reconocen como una característica que describe la práctica de sus docentes, la habilidad de sus profesores para describir de manera clara la dinámica o forma de trabajo así como los criterios que serán tomados en cuenta en el proceso de evaluación, de tal manera que el estudiante tiene claridad acerca de lo que el profesor espera que haga para mostrar el nivel de dominio de una competencia. De la misma forma, reconoce en sus profesores su capacidad de planificar sus clases evitando la improvisación y/o la inversión de esfuerzo sin cumplir los objetivos académicos.

Sin embargo con respecto a las estrategias de aprendizaje implementadas, los estudiantes declaran que sus profesores diseñan tareas y/o actividades poco contextualizadas con la realidad o el medio que lo rodea, de la misma forma, recurren muy poco a la evaluación diagnostica para ubicar lo que el alumno conoce del tema a abordar. El estudiante comenta la falta de pertinencia en las actividades elegidas para un aprendizaje significativo ya que no recurren a proyectos que partan del planteamiento y el análisis de problemáticas reales.

De los resultados mostrados se infiere que los profesores saben planificar su clase, ya que organizan sus actividades y anticipan las criterios a evaluar, esto hace que el estudiante conozca lo que demanda el maestro para obtener una calificación, sin embargo ello no le asegura un aprendizaje significativo, ya que el profesor no muestra competencia en el diseño de estrategias que retomen el contexto del estudiante a partir de la evaluación diagnóstica, de tal manera que pueda vincular las tareas con las situaciones cotidianas que viven los estudiantes, para hacerle sentir que lo que aprende es realmente útil y aplicable a su vida. En este sentido, la función de la estrategia de aprendizaje que el docente diseña es despertar el interés de aprender, de tal manera que el estudiante pueda organizar e integrar el nuevo conocimiento generando la metacognición, para ello se debe aspirar a que los conocimientos no sean solo acumulativos sino prácticos y aplicables, y de esta manera significativos. Fingerman (2010) explica que los aprendizajes significativos logran obtener mayor comprensión sobre los conocimientos adquiridos, por un tiempo prolongado, pues no es una anexión arbitraria sino que los nuevos aprendizajes se suman a los ya existentes de un modo reflexivo y relacionado, dentro de la estructura cognitiva.

De esta manera para lograr que el alumno aprenda significativamente hay que indagar sobre sus ideas previas, motivarlo hacia la conveniencia de no limitarse a una repetición memorística, y presentarles un material potencialmente de interés, que el alumno debe estar dispuesto a analizar y descubrir mediante el anclaje de su propio contexto. El alumno en cuestión del estudio mostró 
la necesidad de que el docente se habilite en el diseño de estrategias y la selección de materiales que sean pertinentes con los intereses de quien aprende y así orientar al estudiante a la búsqueda de sus propias estrategias.

\section{INFRAESTRUCTURA Y LAS ESTRATEGIAS DE APRENDIZAJE}

Se muestra en este apartado la relación existente entre la disponibilidad de las herramientas y recursos y la implementación de las estrategias utilizadas por los docentes para generar el clima escolar.

Tabla 1. Valores correlacionales de "Las herramientas y recursos y las estrategias de aprendizaje.

\begin{tabular}{|l|c|c|c|}
\hline \multirow{2}{*}{\multicolumn{1}{|c|}{ Estrategias de aprendizaje }} & \multicolumn{3}{|c|}{ Recursos y herramientas } \\
\cline { 2 - 4 } & $\begin{array}{c}\text { Calidad de } \\
\text { Espacios } \\
\text { Deportivos }\end{array}$ & $\begin{array}{c}\text { Disponibilidad } \\
\text { de Recursos }\end{array}$ & $\begin{array}{c}\text { Pertinencia y } \\
\text { Disponibilidad } \\
\text { de las TIC }\end{array}$ \\
\hline Manejo de ejemplos reales & .22 & .35 & .46 \\
\hline Motivación a construir conocimiento & .25 & .36 & .43 \\
\hline Tareas contextualizadas & .30 & .34 & .42 \\
\hline Impulso al trabajo de equipo & .30 & .33 & .34 \\
\hline $\begin{array}{l}\text { Clarificación de forma de trabajo y forma de } \\
\text { evaluación }\end{array}$ & .27 & .37 & .31 \\
\hline Planificación de actividades de estudio & .24 & .26 & .21 \\
\hline
\end{tabular}

Fuente: Elaboración propia.

De acuerdo a lo mostrado en la Tabla 1, los jóvenes que reportan que la institución les brinda los espacios adecuados, incluyendo áreas para la práctica del deporte, son los jóvenes que también reportan satisfacción de las estrategias de sus docentes, así, los estudiantes que afirman que la escuela proporciona el uso de herramientas tales como sitios web, videos, libros y artículos actualizados e implementos tecnológicos de acceso a la información, son los estudiantes que reportan estar satisfechos del clima de aprendizaje generado por el uso de estrategias colaborativas como parte del ejercicio docente.

Para los jóvenes, la disponibilidad de recursos y herramientas determina la posibilidad de diseñar estrategias de aprendizaje asertivas y con ello tener un mejor desempeño en el desarrollo de sus competencias. Los jóvenes expresaron su inconformidad ante una exigencia al cumplimiento de una actividad cuando la institución no les proporciona las herramientas y los recursos para su realización. 
Se puede inferir que en la medida en que la institución atiende y gestiona la disposición de las herramientas tecnológicas, instalaciones adecuadas, y espacios para el deporte, los profesores se sienten con las condiciones para utilizar estrategias didácticas participativas y pertinentes para el desarrollo de competencias. Al mismo tiempo que el joven amplía sus posibilidades de interacción con la experiencia y el acto del saber. Al estudiar las relaciones entre infraestructura escolar y resultados académicos Duarte, Gargiulo y Moreno (2011) en las pruebas del Segundo Estudio Regional Comparativo y Explicativo SERCE, se observa que los factores que están más alta y significativamente asociados con los aprendizajes son la presencia de espacios de apoyo a la docencia (bibliotecas, laboratorios de ciencias y salas de computo) la conexión a servicios públicos de electricidad y telefonía y la existencia de agua potable, desagüe y baños en número adecuado. En las escuelas urbanas, además de los anteriores, la presencia de áreas de uso múltiple (gimnasio y/o auditorio) y de espacios para enfermería o servicios psicopedagógicos están asociados con mejores aprendizajes de los estudiantes.

Osorio y Duarte (2011) hablan de los ambientes híbridos de aprendizaje para referirse a aquéllos que combinan la instrucción cara a cara con instrucción mediada por las tecnologías de la información y la comunicación, al respecto consideran que esta combinación optimiza ambos ambientes favoreciendo y fortaleciendo aún más la interacción social y los ambientes colaborativos.

El aprovechamiento de los espacios, los recursos y sus elementos para apoyar directa o indirectamente los ambientes escolares, permite y facilita las interacciones entre los alumnos y el maestro, de tal manera que la estrategia se convierte en una situación de aprendizaje que el maestro puede producir.

\section{COMPARACIONES CORRESPONDIENTES A LA PERCEPCIÓN DE LOS AMBIENTES DE APRENDIZAJE DE LOS JÓVENES}

Para establecer comparaciones entre los grupos se utilizó la prueba paramétrica T-Student, en la idea de que mediante el análisis de las medias considerando su nivel de probabilidad, se establezcan las diferencias o concordancias entre los diversos comportamientos partiendo de la hipótesis de igualdad.

Se presentan a continuación aquellas variables en las que se mostró significancia en su diferencia. 
Tabla 2. Comparaciones de ambientes de aprendizaje por turno.

\begin{tabular}{|l|c|c|c|c|}
\hline \multicolumn{2}{|c}{$\begin{array}{c}\text { PERCEPCIÓN DE LOS AMBIENTES DE APRENDIZAJE DE ALUMNOS DEL } \\
\text { TURO MATUTINO Y VESPERTINO }\end{array}$} \\
\cline { 2 - 4 } \multicolumn{1}{|c|}{ Variable } & Turno del estudiante & Valor & \multirow{2}{*}{$p$} \\
\cline { 2 - 4 } & $X$ Matutino & $X$ Vespertino & $T$ & \\
\hline Comunicación con profesores & 7.84 & 7.28 & 2.084 & 0.038 \\
\hline Búsqueda de apoyo & 7.47 & 6.90 & 2.028 & 0.044 \\
\hline Exigencia a cumplir las reglas & 8.89 & 7.87 & 3.943 & 0.000 \\
\hline Comunicación de reglas & 8.57 & 7.66 & 3.463 & 0.001 \\
\hline Disponibilidad de recursos & 8.10 & 7.31 & 2.547 & 0.012 \\
\hline Pertinencia y disponibilidad de las TICS & 7.28 & 6.59 & 2.068 & 0.040 \\
\hline Manejo de ejemplos reales & 8.23 & 7.28 & 3.512 & 0.001 \\
\hline Motivación a construir conocimiento & 7.61 & 6.85 & 2.500 & 0.013 \\
\hline Impulso al trabajo en equipo & 7.67 & 7.03 & 2.275 & 0.024 \\
\hline Clarificación de forma de trabajo y & 8.43 & 7.82 & 2.561 & 0.011 \\
evaluación & & & & \\
\hline Puntualidad de profesores & 7.51 & 6.91 & 2.064 & 0.040 \\
\hline Planificación de las actividades de estudio & 8.27 & 7.66 & 2.546 & 0.012 \\
\hline Evaluación formativa & 8.07 & 7.55 & 2.206 & 0.028 \\
\hline Evaluación sumativa & 8.02 & 7.27 & 2.389 & 0.018 \\
\hline
\end{tabular}

Fuente: Elaboración propia.

Como se puede observar en la Tabla 2, los estudiantes del turno matutino, expresan mayor comunicación con sus profesores, por lo que tienden a buscar más su apoyo cuando lo necesitan que los alumnos del turno vespertino, declaran además que los profesores tienen mejor manejo de estrategias, al utilizar en sus clases ejemplos reales, por lo que inciden más a la motivación para la construcción del conocimiento que los del turno vespertino. Son más puntuales y se observa que planifican las actividades de estudio, por lo que se refleja mayor diversidad en la forma de evaluar al utilizar más la evaluación formativa y sumativa, por lo que sienten que sus profesores son más justos, pues perciben que usan mejores estrategias de aprendizaje y evaluación. De esto se puede inferir que la relación pedagógica maestro-alumno se ha podido establecer mejor en los jóvenes del turno matutino que en los del vespertino, generando mejores condiciones de acercamiento y confianza con los profesores.

Por otra parte, en cuanto al ambiente institucional, los alumnos del turno matutino declaran que sus autoridades están más al pendiente del cumplimiento de las reglas que el caso de los alumnos del turno vespertino, así mismo se sienten con mejores condiciones de infraestructura al tener 
mayor disponibilidad de recursos, así como contar con más disponibilidad para el uso de tecnología necesaria para su desempeño académico.

Es destacable que las diferencias significativas encontradas fueron siempre a favor de los estudiantes del turno matutino de lo que podemos inferir que los estudiantes de este turno perciben un mejor ambiente de aprendizaje que los del turno vespertino, esto sugiere que se sienten mejor atendidos por la estructura organizacional de la escuela, por lo que las condiciones de trabajo y de aprendizaje son mejores.

Tabla 3. Comparaciones de ambiente de aprendizaje por turno.

\begin{tabular}{|l|c|c|c|c|}
\hline \multicolumn{3}{|c|}{$\begin{array}{c}\text { PERCEPCIÓN DE LOS AMBIENTES DE APRENDIZAJE DE ALUMNOS DEL } \\
\text { TURNO MATUTINO Y NOCTURNO }\end{array}$} \\
\cline { 2 - 5 } \multicolumn{1}{|c|}{ Variable } & \multicolumn{2}{|c|}{ Turno del estudiante } & Valor & Valor \\
& $X$ Matutino & $X$ Nocturno & $T$ & $p$ \\
\hline Búsqueda de apoyo & 7.47 & 6.58 & 2.155 & 0.033 \\
\hline Calidad de relación con el director & 5.32 & 3.97 & 2.223 & 0.028 \\
\hline Confianza en el director & 6.06 & 4.45 & 2.694 & 0.008 \\
\hline Exigencia a cumplir las reglas & 8.89 & 6.37 & 7.236 & 0.000 \\
\hline Comunicación de reglas & 8.57 & 7.47 & 2.985 & 0.003 \\
\hline Castigo al rompimiento de reglas & 7.92 & 5.97 & 4.287 & 0.000 \\
\hline Daño a las instalaciones & 8.03 & 6.53 & 3.092 & 0.002 \\
\hline
\end{tabular}

Fuente: Elaboración propia.

La Tabla 3 muestra las diferencias en las percepciones que tienen los estudiantes de los tunos matutino y nocturno, habiéndose encontrado éstas en las siguientes variables:

Los jóvenes del turno matutino tienen mejor relación y confianza con su director y tienden a buscar más apoyo con sus maestros que los del turno nocturno, de lo que se infiere que su ambiente de aprendizaje en lo referido a la relación pedagógica maestro-alumno y relación con las autoridades es mejor.

En relación a la normatividad de la escuela los jóvenes del turno matutino perciben que es mejor que los del turno nocturno cuando manifiestan que se les ponen muy en claro las reglas escolares, se les exige que las cumplan y se les castiga si no las cumplen. Sin embargo perciben, que a pesar de esto, dañan las instalaciones y/o el mobiliario (graffiti, rayones, etc.) más que los del turno nocturno. De esto se puede inferir que aunque hay mayor atención y vigilancia al cumplimiento de reglas, hay también mayor tendencia a infringirlas que el turno nocturno. 


\section{DISCUSIÓN}

Tal como lo plantean Bernheim (2011), la interacción social produce un favorecimiento del aprendizaje mediante la creación de conflictos cognitivos que causan un cambio conceptual, la socialización entre los alumnos ayuda en la construcción de un conocimiento mediado que cambia el esquema del individuo, por ello se propone como primer principio para un ambiente de aprendizaje constructivista: facilitar que todas las personas del grupo se conozcan para crear un clima cohesionado con objetivos y metas comunes.

Así pues, en este estudio se muestra a partir de los datos obtenidos, que una característica muy importante del ambiente de aprendizaje es el tipo de relación que se establece entre los jóvenes estudiantes, identificándose por un elevado compañerismo y amistad, demostrando además tener una apropiada comunicación y confianza en sus profesores, sin embargo, también se evidenció un distanciamiento con las autoridades de la institución escolar, quienes representan la parte normativa y gestora de condiciones adecuadas para la generación de ambientes de aprendizaje. Vite H. R. (2012) se describe los ambientes de aprendizaje como las condiciones físicas, sociales y educativas en las que se ubican las situaciones de aprendizaje, lo que sugiere el priorizar el clima de las relaciones sociales y considera que un ambiente de aprendizaje se logra cuando todos se sienten cómodos.

Por otra parte, habría que considerar que el estudio estuvo delimitado a una población que pertenece a una sola institución del nivel medio superior, por lo que el contexto fue circunscrito a un tipo específico de programa educativo. Sin embargo, el procedimiento metodológico empleado permitió conocer y analizar a detalle la percepción del estudiante de los ambientes de aprendizaje generados a partir de las condiciones que la institución les brinda incluyendo la interacción social entre todos los actores y las condiciones de infraestructura otorgadas.

Dicho procedimiento podría ser utilizado en investigaciones similares en otros contextos, además los resultados pueden ser generalizados en la medida en que las poblaciones presenten condiciones similares como nivel escolaridad, edad de los sujetos, tipo de subsistema de procedencia entre otros.

A partir de los resultados de este estudio se pueden desarrollar nuevas líneas de investigación enfocadas a describir aspectos específicos que tiene que ver con cada uno de los factores que intervienen en los ambientes de aprendizaje, tales como la interacción social y la práctica docente. 


\section{CONCLUSIONES}

Los jóvenes estudiantes reconocen el desarrollo de sus habilidades sociales como una competencia altamente lograda, lo que implica que se podría construir un clima escolar favorable para promover el aprendizaje colaborativo, ya que muestran disposición a compartir a través de la convivencia mediante valores aceptables para asegurar la armonía en grupo.

Sin embargo, la relación con las autoridades escolares es insatisfactoria y a mayor rango de responsabilidad menor contacto directo hay con los estudiantes. Además para los estudiantes es muy importante que las autoridades de todos los niveles estén en contacto con ellos especialmente el director de la escuela. El contacto con sus autoridades implica la oportunidad de sentirse atendido y sobre todo comprometido en la aplicación de la norma, ello le fortalece además su sentido de pertenencia e identidad con la institución.

Es un clima de sociohabilidad que está poco aprovechado por las autoridades institucionales para orientar las condiciones a la promoción de ambientes de aprendizaje apoyados inclusive en las tecnologías, factor que los estudiantes demandan como una de sus principales necesidades.

En cuanto a la normatividad, se puede inferir que los estudiantes se dan cuenta que no siempre cumplen con las reglas que rigen en su escuela, ya que éstas son sentidas más como una imposición de control que como una generación de hábitos pertinentes para su desarrollo escolar, lo que implica el reto al desacato.

Con respecto a la práctica docente, reconoce que los profesores saben planificar su clase, en lo que refiere a la habilidad para organizar y planificar las actividades docentes, incluyendo la práctica de la evaluación de los aprendizajes, sin embargo ello no le asegura un aprendizaje significativo, ya que, los profesores no consideran el conocimiento previo de donde el estudiante puede partir para el desarrollo de nuevos aprendizajes y competencias, y por otra parte la habilidad de vincular las tareas con las situaciones cotidianas que viven los estudiantes, es decir diseñar estrategias que permitan darle sentido y considerar el contexto .

En las necesidades de infraestructura, los estudiantes, demandan de condiciones en las que pueda socializar y ejercer actividades físicas donde pueda mejorar su calidad de vida, además de tener una opción para el disfrute personal y fortalecer de manera sana y asertiva su relación con los demás (tecnologías y espacios deportivos/convivencia).

De acuerdo a lo anterior, se puede inferir que en la medida en que la institución atiende y gestiona la disposición de las herramientas tecnológicas, instalaciones adecuadas, y espacios para 
el deporte, los profesores se sienten con las condiciones para utilizar estrategias didácticas participativas y pertinentes para el desarrollo de competencias.

Con respecto a percepción de los jóvenes en los diferentes turnos, los estudiantes del turno matutino perciben un mejor ambiente de aprendizaje que los del turno vespertino y nocturno, esto sugiere que se sienten mejor atendidos por la estructura organizacional de la escuela, por lo que las condiciones de trabajo y de aprendizaje son mejores.

\section{Bibliografía}

Bernheim, C. T. (2011). El constructivismo y el aprendizaje de los estudiantes. Universidades, 61(48), 21-32.

Biggs, J. (2006). Calidad del aprendizaje universitario. $2^{\text {a }}$ Edición. España: Narcea.

Bolaños, B. G., Molina, B. Z. (2007). Introducción al currículo. 21 ed. Costa Rica: Universidad Estatal a Distancia.

Caso, J., Salgado, B., Rodríguez, J. C., Contreras, L. A. y Urías, E. (2010). Propiedades psicométricas de la Escala de Clima Escolar para adolescentes. UEE RT 10 - 002. Ensenada, México. Universidad Autónoma de Baja California.

Dueñas, G. (2015). La normatividad escolar: orden, disciplina y derechos humanos. Revista de Educación y cultura az. http://educacionyculturaaz.com/analisis/lanormatividad-escolarorden-disciplina-y-derechos-humanos.

Duarte J., Gargiulo C., Moreno M. (2011). Infraestructura escolar y aprendizaje en la educación básica latinoamericana: Un análisis a partir del SERCE. BID.

Fingermann H. (2010). Estrategias para el aprendizaje significativo. La Guía de Educación http://educacion.laguia2000.com/estrategias-didacticas/estrategias-para-el-aprendizajesignificativo\#ixzz4XO7hMqW1

Gimeno, S.J., Pérez, G. A. (2008). La enseñanza: su teoría y práctica. 6th ed. España: Ediciones Akal.

Medina, M. B. E. (2015). Influencia de la interacción alumno-docente en el proceso enseñanzaaprendizaje. Paakat: Revista de Tecnología y Sociedad, (8). 
Ortiz, S., Giraldo, O. (2009). Los ámbitos de interacción social en la formación de competencias laborales generales en los estudiantes de la básica secundaria. Universidad de Manizales. CINDE. Colombia.

Osorio, G. L. A. (2011). Interacción en ambientes híbridos de aprendizaje. 1st ed. España: UOC.

Parra, R. J. (2007). Aprendizaje y conectividad. 1st ed. Colombia: Javegraf.

RIEMS. (2008). Reforma Integral de Educación Media Superior. Diario Oficial de la Federación. México.

Rodríguez, V. (2014). Ambientes de Aprendizaje. Revista Ciencia Huasteca, Vol. 2 No. 4.

Tobón, S. T., Prieto, J. H. P., \& Fraile, J. A. G. (2010). Secuencias didácticas: aprendizaje y evaluación de competencias. México: Pearson educación.

Vite, H. R. (2012). Ambientes de Aprendizaje. Universidad Autónoma del Estado de Hidalgo. Disponible en: http://www.uaeh.edu.mx/scige/boletin/huejutla/n4/e1.html 